Article

\title{
Usefulness of a titratable mandibular positioner during sleep endoscopy as a prediction tool for treatment outcome with mandibular advancement devices: DISE-SAM protocol.
}

\author{
Patricia Fernández-Sanjuán ${ }^{1,2}$, Juan José Arrieta ${ }^{3}$, Jaime Sanabria4, Marta Alcaraz ${ }^{5}$, Gabriela Bosco ${ }^{5,6}$, Nuria Pérez- \\ Martín' ${ }^{5,6}$, Adriana Pérez ${ }^{7}$, Marina Carrasco-LLatas ${ }^{8}$, Isabel Moreno-Hay ${ }^{9}$, Marcos Ríos-Lago ${ }^{10}$, Rodolfo Lugo ${ }^{11}$, Car- \\ los O'Connor-Reina $^{12,13}$, Peter Baptista ${ }^{14}$, Guillermo Plaza ${ }^{5,6}$,*
}

1 Department of Maxillofacial Surgery and Dentistry, Hospital Universitario San Francisco de Asís, 28002, Madrid, Spain. Universidad Rey Juan Carlos (P.F-S.).

2 Dental Sleep Medicine, Hospital Universitario Sanitas La Zarzuela, 28942 Madrid, Spain.

3 Department of Stomatology, Hospital Universitario Fundación Jiménez Díaz, 28040 Madrid, Spain. (JJ.A.)

4 Department of Otolaryngology Head and Neck Surgery, Hospital Universitario Fundación Jiménez Díaz, 28040 Madrid, Spain. (J.S.)

5 Department of Otolaryngology Head and Neck Surgery, Hospital Universitario Sanitas La Zarzuela, 28942 Madrid, Spain. (M.A.)

6 Department of Otolaryngology Head and Neck Surgery, Hospital Universitario de Fuenlabrada, 28942 Madrid, Spain. Universidad Rey Juan Carlos (G.B.; N.P.-M.; G.P.).

7 Department of Otolaryngology Head and Neck Surgery, Hospital La Milagrosa, 28010 Madrid, Spain. (A.P.)

8 Department of Otolaryngology Head and Neck Surgery, Hospital Universitario Dr. Peset. 46017 Valencia, Spain. (M.C.-Ll)

9 College of Dentistry, University of Kentucky, 40536 Lexington, Kentucky, USA. (I.M.-H.)

10 Department of Basic Psychology II. UNED. 28040, Madrid, Spain. (M.R.-L.)

11 Department of Otolaryngology Head and Neck Surgery, Hospital San José, 64718 Monterrey, México. (R.L.)

12 Department of Otolaryngology Head and Neck Surgery, Hospital Quironsalud Marbella, 29603 Marbella, Spain. (C.O.)

13 Department of Otolaryngology Head and Neck Surgery, Hospital Quironsalud Campo de Gibraltar, 11379 Palmones, Spain.

14 Department of Otolaryngology Head and Neck Surgery, Clínica Universitaria de Navarra, 31008 Pamplona, Spain. (P.B.)

* $\quad$ Correspondence: gplaza.hflr@salud.madrid.org

\begin{abstract}
Mandibular advancement devices (MAD) are an effective alternative treatment to CPAP. Different maneuvers have been performed during drug sleep-induced endoscopy (DISE) to mimic the effect of MAD. Using the Selector Avance Mandibular (SAM) device, we aimed to identify MAD candidates during DISE using a titratable, reproducible, and measurable maneuver. This DISE-SAM protocol may help to find the relationship between the severity of the respiratory disorder and the degree of response, and to determine the advancement required to improve the collapsibility of the upper airway. Explorations were performed in 161 patients (132 males; 29 females) with a mean age of 46.81 $(\mathrm{SD}=11.42)$ years, a BMI of $27.90(\mathrm{SD}=4.19) \mathrm{kg} / \mathrm{m} 2$ and a mean $\mathrm{AHI}$ of $26.51(\mathrm{SD}=21.23)$. Results showed no relationship between severity and MAD recommendation. Also, there was a weak positive relationship between the advancement required to obtain a response and the disease severity. Using the DISE-SAM protocol, the response and the range of mandibular protrusion were assessed, avoiding the interexaminer bias of the jaw thrust maneuver. We suggest prescribing MAD as a single, alternative, or multiple treatment approaches following the SAM recommendations in a personalized design.
\end{abstract}

Keywords: sleep breathing disorders; mandibular advancement; MAD; titratable positioner; drug-induced sleep endoscopy; patient selection. 


\section{Introduction}

Obstructive sleep apnea (OSA) is a highly prevalent disease, with significant public health outcomes [1] whose primary symptom is excessive daytime sleepiness that, together with mood alteration and cognitive impairment, produce a progressive deterioration in the quality of life of patients. In addition, it has been associated with an increased risk of arterial hypertension, cardiovascular morbidity and mortality, and occupational and traffic accidents [2,3].

Although continuous positive air pressure (CPAP) is considered the gold standard treatment for OSA, it is estimated that failure to CPAP occurs in approximately 35\% of patients [4] and that 29 to $83 \%$ of users are nonadherent to this therapy [5]. Mandibular advancement devices (MAD) are an effective alternative treatment to CPAP for many of these patients. Their mechanism of action is based on the advancement and stabilization of the mandible and hyoid. They increase basal electromyographic activity of the genioglossus, tighten the palatoglossus and palatopharyngeus muscles, increase the vertical dimension and activate the lateral walls of the velopharynx [6-10]. Also, the soft palate is displaced ventrally increasing the retromandibular space's caliber [6,7]. MAD are indicated in patients with simple snoring, mild and moderate OSA without comorbidity and severe OSA patients who refuse or do not tolerate CPAP [11]. Although CPAP is more effective in reducing the Apnea Hypoapnea Index (AHI), MAD is preferred by patients and their partners as they are more portable, make no noise, are more comfortable, and better tolerated $[12,13]$. However, individual variability exists in response to MAD treatment [14]. Some studies show that MAD are effective in 30-81\% [15]. This high variability in response suggests the need for the identification of potential responders.

There are numerous studies aimed at identifying suitable candidates for treatment with MAD on occasions with contradictory results. Despite it is accepted that individuals with less severe and predominantly supine OSA respond better [16,17], some publications find MAD a good alternative also for severe patients [18] and non-positional OSA [14]. Considering anthropometric variables, younger patients with low body mass index or female candidates may also have better responses [19-22]. Cephalometric variables are considered inconsistent predictors and therefore, reliance on them for this purpose is discouraged [23]. Even some values for air pressure have been identified as having very high predictive values in detecting MAD non-responder patients [24-26]. Regarding polysomnographic variables, some studies agree that MAD therapy is more effective in nonREM related OSA [14]. Considering the multifactorial origin of OSA [27], a more significant response to oral therapy occurred in those patients with a mild anatomical compromise and a lower loop gain [28,29]. Finally, Sutherland et al. [30] performed a study on awake multimodal phenotyping to predict MAD treatment outcome. They found that it does not enhance outcome prediction, concluding that office-based awake assessments have limited utility for robust clinical prediction models and suggested that future work should focus on sleep-related assessments. However, due to the growing interest in MAD as a therapeutic option and knowing that OSA is not adequately alleviated in all patients wearing MAD, it is mandatory to find an objective and reproducible procedure to help in the patient selection.

To date, drug-induced sleep endoscopy (DISE) provides the most helpful information on upper airway (UA) collapsibility during sleep compared to other evaluation techniques available [31, 32]. DISE was first described by Croft and Pringle [33] and has become widely used. It involves the assessment of the UA by means of fiberoptic endoscopy under pharmacologic sedation, which simulates sleep. Nowadays, this procedure is performed in patients seeking alternatives to CPAP treatment. To mimic the effect of MAD, different maneuvers have been used. For example, it has been shown that the results obtained by performing manually jaw thrust maneuvers do not always correlate with those achieved by intraoral appliances [34-37]. The possible cause of this difficulty could be inconsistency in the application of the maneuver, inaccurate measurement of the advancement in some cases, or patient stimulation that could lead to arousals [38]. 
The aim of the present study is twofold. First, to identify those patients during DISE who will potentially benefit from oral appliance therapy using a titratable, reproducible, and measurable maneuver, with the Selector Avance Mandibular (SAM) device. Second, to study the relationship between the severity of the respiratory disorder and the degree of response and the degree of advancement required to respond during DISE. And in addition, it may also suggest different levels of recommendation to optimize MAD treatment in a personalized design according to the expected response.

\section{Materials and Methods}

\section{Participants}

A prospective study was designed. The Medical Ethical Committee of Hospital Universitario de Fuenlabrada approved the study protocol in accordance with the Declaration of Helsinki (APR 20/30). Data on study subjects were collected and stored anonymously to protect personal information.

The study included one hundred and sixty-one patients (132 males; 29 females). The inclusion criteria were adults ( $>18$ years) with snoring and OSA. Patients were evaluated by standard full night polysomnography and interpreted using the diagnostic criteria established by the American Academy of Sleep Medicine [39]

Exclusion criteria were propofol allergy, periodontal disease, insufficient number of teeth, acute or severe temporomandibular disorder or an exaggerated gag reflex. Patients with medical history of congenital abnormalities of the UA were also excluded.

\section{Methods}

The study protocol included a complete medical history, with the following variables: age, sex, body mass index (BMI), and Epworth Sleepiness Scale (ESS) score. All patients underwent diagnostic sleep polysomnography (PSG). All patients underwent an office examination including awake UA endoscopy examination to identify anatomical alterations. Orthopantomography, periodontal, dental, and muscular/articular examinations were also performed in the dental office to confirm the patient's suitability to use MAD.

Subsequently, the anesthetic assessment was done prior to DISE. The procedure was performed in an operating theatre as a day-case technique. The clinicians involved in the procedure included an anesthesiologist, an otolaryngologist, and a dentist. A flexible video endoscope (TGH Endoscopia. MACHIDA ENT-30PIII, Madrid Spain) was used to visualize the UA. The classification system used to evaluate the UAs patterns and areas of collapse was the VOTE scale [40].

The DISE-SAM protocol was then started. To mimic a mandibular advancement splint therapy, it incorporates a titratable tool, so called Selector Avance Mandibular (SAM), that has been designed to produce personalized mandibular advancement during DISE by the first author. In addition, it allows the operator to manually perform controlled, progressive, and high precision advancements (in the range of millimeters) during sedation, starting from no advancement position until reaching the optimal position in which, if patient responds, apneas/snoring are reduced or eliminated (Figure 1 and Video). 


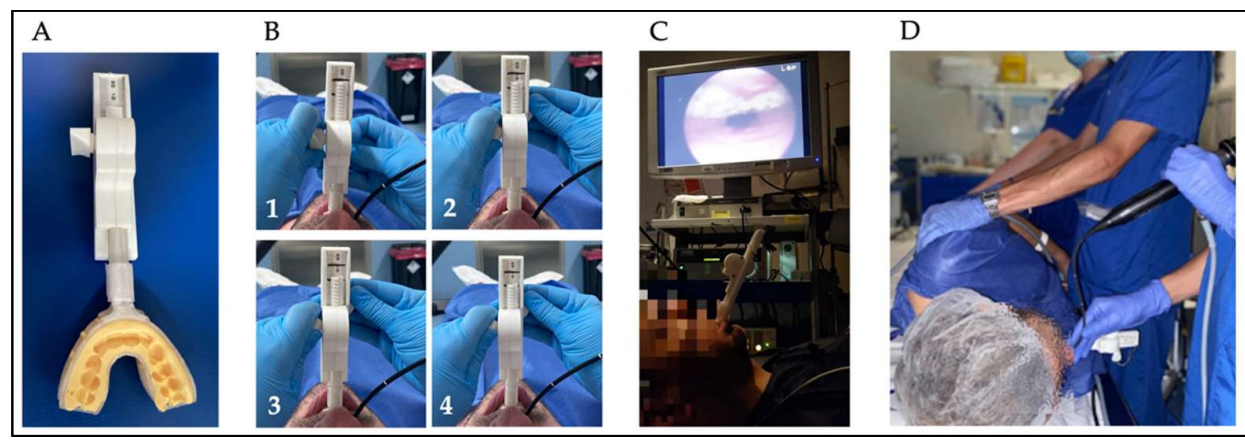

Figure 1: A) SAM device. B) SAM titration (1-4): 0\%, 50\%, 75\% and 100\% advancement respectively. C) Patient examination in supine position. D) Patient examination in lateral decubitus position.

Before starting the sedation, the SAM was personalized to the patient's dental arches with polyvinylsiloxane dental impression material. While the patient is awake, the maximum retrusive and protrusive positions were recorded by using a measuring scale where the complete range of anteroposterior jaw movement is delimited. During sedation, mandibular movement was limited to this previous measurement to reproduce the natural and tolerable jaw movement. Following the European position paper recommendations on DISE [31], propofol was used in this study. The drug delivery system was target-controlled infusion. During the procedure levels between 50 and 70 (bispectral index-BIS) should be maintained. No vasoconstrictors or anesthetics were used. The fiberscope was introduced and placed in the nasopharynx where the operator waited until the appearance and visualization of two cycles of snoring, apnea and breathing. Then, UA was explored up to the larynx analyzing the behavior at the different VOTE levels. The neck and /or trunk was moved to lateral decubitus to assess positional therapy [41].

Once the UA was studied in the patient's baseline situation, the SAM customized to the dental arches was introduced in the patients' mouth and evaluated the mandibular advancement positions for $0 \%, 50 \%, 75 \%$ and 100\% consecutively (Figure 1 ). The locations of the obstructions, the degree and pattern of collapse were carefully noted. When a good response was found, slow descent was done until the minimum effective advancement was reached. Once the minimum effective position in supine was found in the responders, the examination was performed in lateral decubitus. Thus, the assessments were performed in supine position and lateral head or trunk rotation with jaw advancement to evaluate the effect of a MAD, the effect of positional therapy, and to mimic combined treatment (positional therapy plus MAD) (Figure 2).

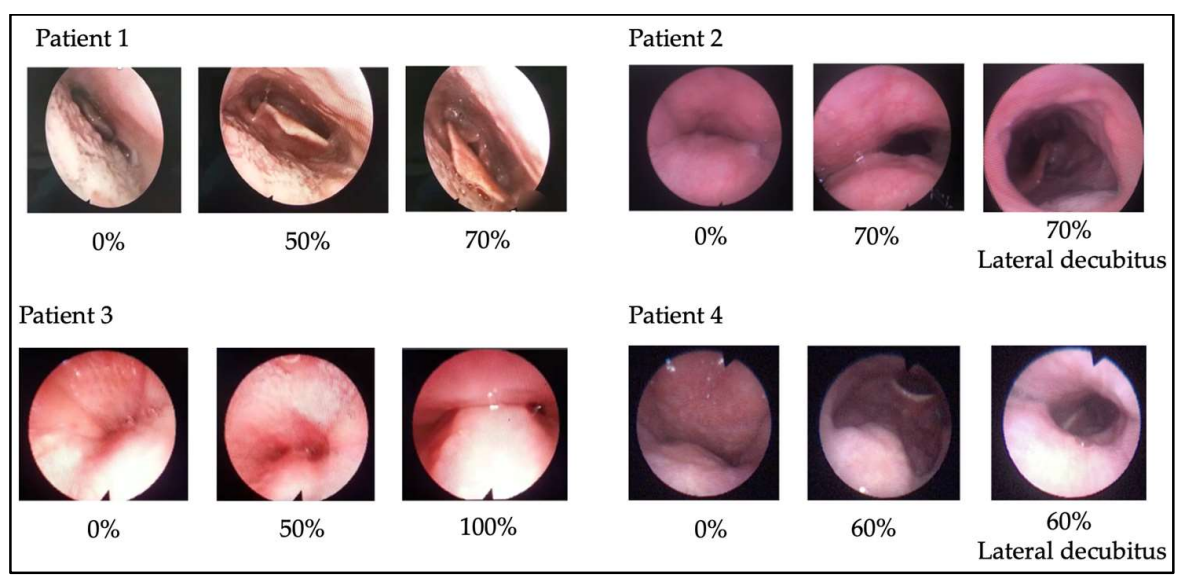

Figure 2: Behavior of the UA when introducing advancements and body position maneuvers. Patient 1: UA is analyzed with $0 \%-50 \%$ and $70 \%$ advancement. At $50 \%$ and $70 \%$ an effective response is seen. Patient 2 : UA is analyzed with $0 \%-70 \%$ and $70 \%$ plus right lateral decubitus. The combination of MAD + positional therapy significantly improves collapsibility. Patient 3: UA is analyzed with $0 \%-50 \%$ and $100 \%$ advancement. Despite transforming the circular collapse into anteroposterior collapse in velopharynx, the advancement is not effective. 
Patient 4: UA is analyzed with $0 \%-60 \%$ and $60 \%$ plus right lateral decubitus. Advancement to $60 \%$ is very effective in supine position at velopharynx but in right lateral decubitus an epiglottis collapse appears.

A valid response was considered when the patient remained at least 3 minutes without apneas and controlled $\mathrm{O}_{2}$ saturation. Once the response to the jaw advancement was visualized both in supine and lateral decubitus, the SAM was withdrawn. Only one examination could not be completed because of sneezing, coughing and excessive expectoration.

A scale of four levels of MAD recommendation was used, depending on the degree of response and the need or not for combined treatment. These four levels are described in Table 1.

Table 1: SAM Recommendations (Single use - Alternative treatment - Multiple treatment approach. *Greater predictive value in young patients, BMI $<30$, without predominance of apneas in REM sleep and with predominance of obstructive hypopneas vs apneas in sleep study.

\begin{tabular}{|c|c|}
\hline $\begin{array}{c}\text { S Recommendation } \\
\text { (Single use) }\end{array}$ & $\begin{array}{l}\text { As a single treatment if collapse }(1 \text { and/or } 2) \text { was eliminated at all levels } \\
\text { VOTE (0000) for patients with simple snoring or OSA. * }\end{array}$ \\
\hline $\begin{array}{l}\text { A Recommendation } \\
\text { (Alternative treatment) }\end{array}$ & $\begin{array}{l}\text { As an alternative treatment if the collapses were improved by at least } 50 \% \text {, } \\
\text { improving from } 2 \text { to } 1 \text { and from } 1 \text { to } 0 \text {, with some VOTE levels remaining at } \\
\text { 1. If collapse } 2 \text { persisted at one level, it had to be momentary and shouldn't } \\
\text { be responsible for apneas maintaining normal oxygen saturation. Snoring is } \\
\text { expected due to persistent vibration and partial collapse at some level. The } \\
\text { decrease in AHI is expected to be less than in S recommendation. Results } \\
\text { may improve with therapy combination (surgical, positional therapy, myo- } \\
\text { functional therapy...). If the patient refuses any combination of treatments } \\
\text { to improve the outcome, an improvement from baseline is expected. Partial } \\
\text { response expected. * }\end{array}$ \\
\hline $\begin{array}{c}\text { M Recommendation } \\
\text { (Multiple treatment } \\
\text { approach) }\end{array}$ & $\begin{array}{l}\text { ONLY as a combined treatment if the collapsibility had improved at some } \\
\text { VOTE level but remained value } 2 \text { (even with positional therapy) at any } \\
\text { VOTE level responsible for apneas and coexisted recommendation for up- } \\
\text { per airway surgery. It could be recommended in combination with CPAP to } \\
\text { reduce pressures if this was the cause of poor tolerance. }{ }^{*}\end{array}$ \\
\hline $\begin{array}{l}\text { NO Recommendation } \\
\text { (MAD not recommended) }\end{array}$ & $\begin{array}{l}\text { MAD is not recommended if no change at VOTE levels is visualized, or com- } \\
\text { plete collapses responsible for apneas remained without surgical recom- } \\
\text { mendation or improvement in lateral decubitus. Also, if collapsibility wors- } \\
\text { ened with mandibular advancement. Likewise, if maximum advancement } \\
\text { was needed to achieve significant changes in UA patency due to poor toler- } \\
\text { ance expected. }\end{array}$ \\
\hline
\end{tabular}

\section{Data analyses}

Kolmogorov-Smirnov tests for normality were used to determine whether the distribution of continuous variables (age, IAH, BMI and ESS) fitted the normal distribution in each group ( $p>0.05$ in all cases). Thus, one-way ANOVA was applied to explore the existence of differences among groups in age, BMI, and ESS (Bonferroni correction was applied for post-hoc analyses). A variable "SAM recommendation" was constructed according to the criteria established in the procedure. In addition, a new variable MAD YES/MAD NO was built according to whether MAD was recommended or not. Statistical analyses of categorical variables were performed using the chi-square test. This was applied to study a relationship between severity and gender, "SAM recommendation" and "MAD YES/NO". To explore the relation between severity and advancement percentage 
Sommers' d test was applied. It is an ordinal directional measure that indicates the strength and direction of the relationship between variables. Finally, bivariate Pearson correlations were performed to analyze possible relationships among age, AHI, BMI, and ESS. To study advancement percentage correlations, Spearman rho was applied. A probability value less than 0.05 was considered to indicate the statistical significance and all reported probability values are 2-tailed. Finally, the SPSS v.24.0 statistical software package was used to perform analyses.

\section{Results}

In total, 161 patients were included for analysis. Patients' mean age was $46.82 \pm 11.42$ years; mean BMI was $27.9 \pm 4.19 \mathrm{~kg} / \mathrm{m} 2$, mean AHI was $26.43 \pm 21.23$ events per hour and mean EES was $9.87 \pm 5.49$. Participants were assigned to one of these four groups based on the AHI score as follows: snoring group, AHI 0-4.9 events per hour; mild OSA group, AHI 5-14.9 events per hour; moderate OSA group: AHI 15-29.9 events per hour; and severe OSA group, AHI>30 events per hour. The demographic characteristics of the four severity groups are summarized in Table 2.

Table 2: Statistical properties of the four severity groups

\begin{tabular}{|l|c|c|c|c|}
\hline & Snoring Group & Mild & Moderate & Severe \\
\hline $\mathrm{n}$ & 23 & 36 & 48 & 54 \\
\hline Gender (m/f) & $20 / 3$ & $25 / 11$ & $42 / 6$ & $45 / 9$ \\
\hline Age M (SD) & $42.74(9.65)$ & $46.39(13.12)$ & $46.27(11.72)$ & $49.42(10.22)$ \\
\hline BMI M (SD) & $26.06(3.03)$ & $26.7(3.22)$ & $27.82(3.87)$ & $29.96(4.98)$ \\
\hline AHI M (SD) & $2.49(1.80)$ & $9.01(2.61)$ & $23.28(4.41)$ & $51.28(15.73)$ \\
\hline ESS M (SD) & $8.33(3.52)$ & $9.52(5.49)$ & $9.21(5.45)$ & $11.52(6.09)$ \\
\hline
\end{tabular}

AHI: Apnea Hipopnea Index; BMI: Body Mass Index; ESS: Epworth sleepiness scale, M: mean; SD: standard deviation.

All groups were matched for age $(\mathrm{F}=1.97 ; \mathrm{p}=0.12)$ and $\mathrm{ESS}(\mathrm{F}=1.31 ; \mathrm{p}=0.28)$ but not for BMI ( $\mathrm{F}=5.49 ; \mathrm{p}=0.001)$. Bonferroni's post hoc comparison showed differences in BMI between groups snorer and severe, and mild and severe ( $p=0.005$ and $p=0.007$ respectively). No differences in gender were found among groups (Chi-squared $=5.27 ; \mathrm{p}=0.15$ ).

MAD was not recommended in $26.1 \%$ of the patients. Of the $73.9 \%$ of patients who did receive a recommendation for MAD therapy, 19.3\% received an $S$ recommendation, $34.2 \%$ received an $\mathrm{A}$ recommendation, and $20.5 \%$ received an $\mathrm{M}$ recommendation. Table 3 shows the percentage and number of patients classified according to severity who received the different SAM recommendations (see also Figure 3).

Table 3: Proportion and number of patients classified by severity within each level of recommendation.

\begin{tabular}{|l|l|l|l|l|l|}
\hline & Snorers \% (n) & Mild \% (n) & Moderate \% (n) & Severe \% (n) & Total \% (n) \\
\hline S recommendation & $22,6(7)$ & $29,0(9)$ & $32,2(10)$ & $16,1(5)$ & $100(31)$ \\
\hline A recommendation & $14,5(8)$ & $21,8(12)$ & $38,2(21)$ & $25,5(14)$ & $100(55)$ \\
\hline M recommendation & $12,1(4)$ & $21,2(7)$ & $18,2(6)$ & $48,5(16)$ & $100(33)$ \\
\hline NO recommendation & $9,5(4)$ & $19,0(8)$ & $26,2(11)$ & $45,2(19)$ & $100(42)$ \\
\hline
\end{tabular}




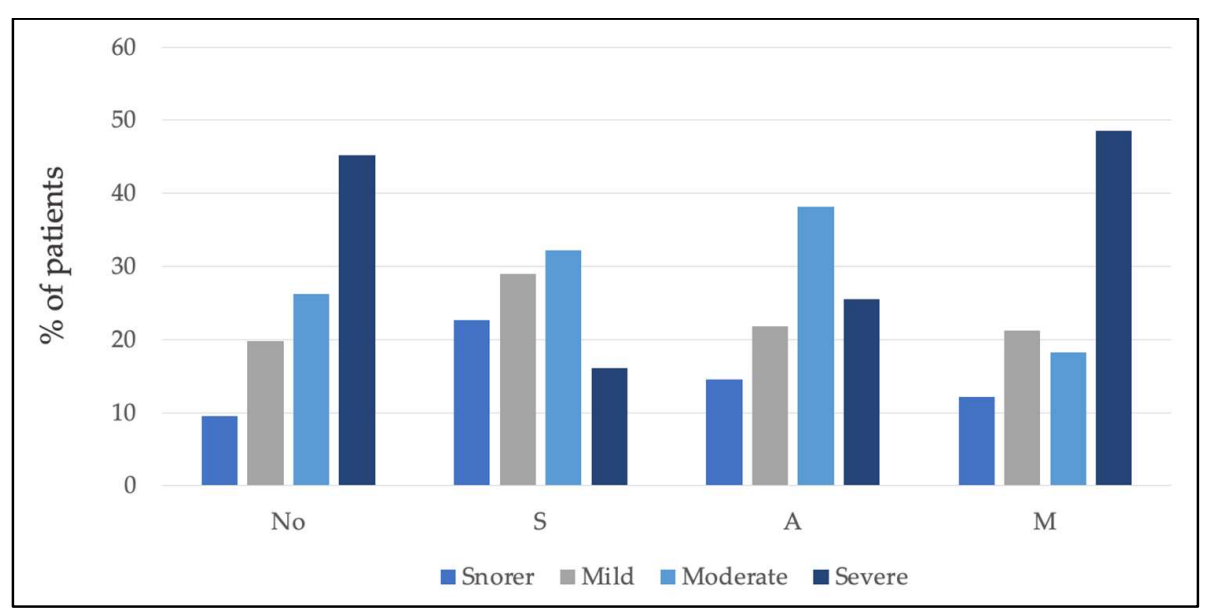

Figure 3: Within each level of recommendation, it is shown the percentage of patients according to severity. Note that the summatory of each recommendation is $100 \%$.

Also, percentages and number of patients in each group of severity receiving different recommendations were calculated (Table 4 and Figure 4).

Table 4: Proportion and number of patients classified by recommendation within each level of severity.

\begin{tabular}{|l|l|l|l|l|l|}
\hline & Snorers \% (n) & Mild \% (n) & Moderate \% (n) & Severe \% (n) & Total \% (n) \\
\hline S recommendation & $30,4(7)$ & $25(9)$ & $20,8(10)$ & $9,3(5)$ & $19,3(31)$ \\
\hline A recommendation & $34,8(8)$ & $33,3(12)$ & $43,8(21)$ & $25,9(14)$ & $34,2(55)$ \\
\hline M recommendation & $17,4(4)$ & $19,4(7)$ & $12,5(6)$ & $29,6(16)$ & $20,5(33)$ \\
\hline NO recommendation & $17,4(4)$ & $22,2(8)$ & $22,9(11)$ & $35,2(19)$ & $26,1(42)$ \\
\hline & & & & & $100(161)$ \\
\hline
\end{tabular}

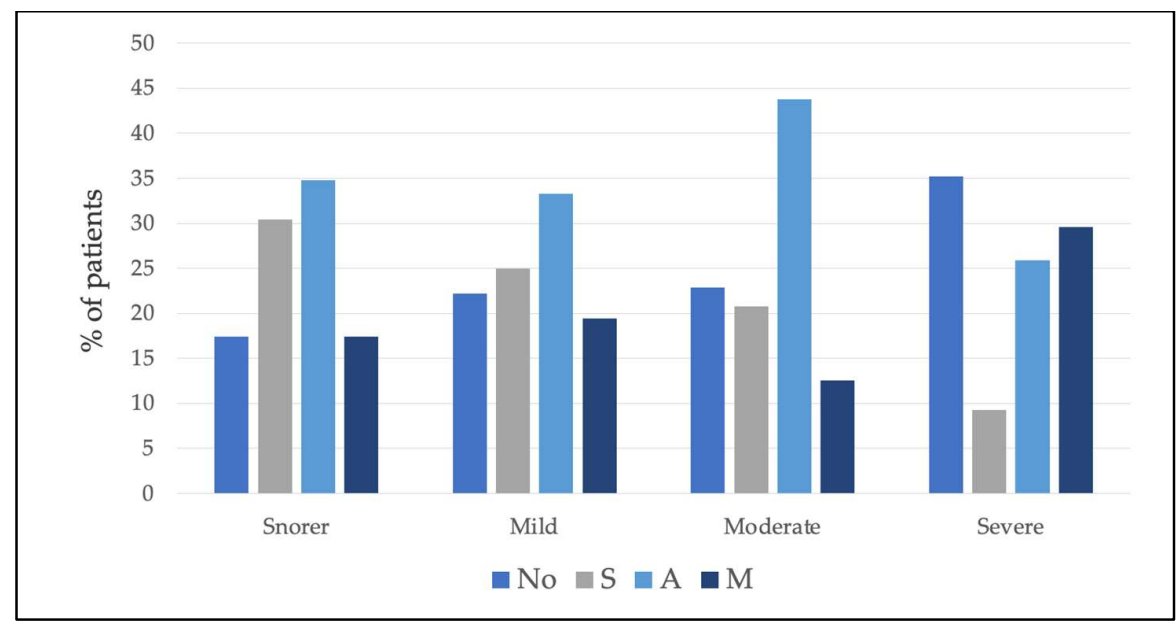

Figure 4: Within each severity group the percentage of patients classified by recommendation is shown. Note that the summatory of each severity level is $100 \%$.

Results showed no relationship between severity and MAD Yes/No (Chisquared $=3.75 ; p=0.29$ ) and SAM recommendation (Chi-squared=13.94; $p=0.12$ ) (see Figure $5)$. 


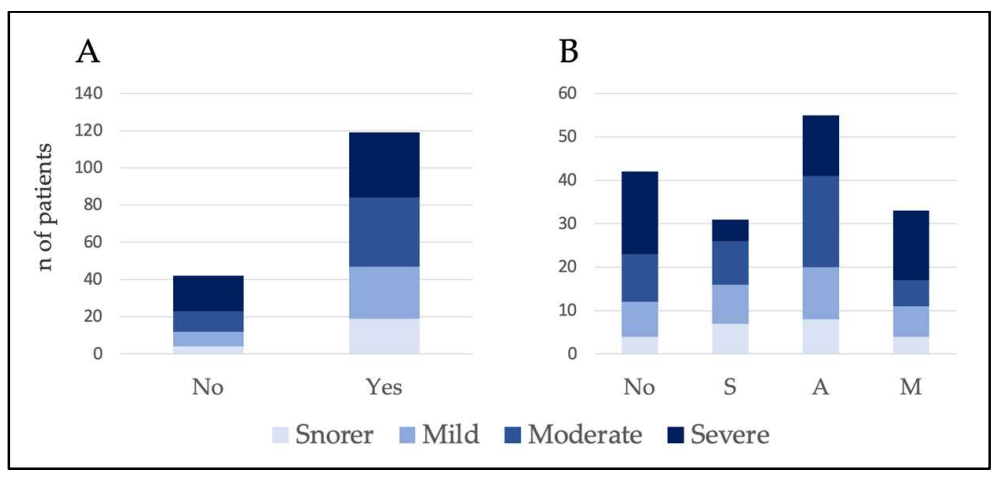

Figure 5: Histograms showing the number of patients for whom the MAD Yes/No recommendation (A), and SAM recommendation (B) were made. No: not recommended; S: single use; A: alternative treatment; M: multiple treatment approach.

Results indicate a positive relationship between severity and advancement percentage $(d=0.15 ; p=0.048)$. Figure 6 shows the necessary mandibular advancement percentage to obtain response during DISE according to severity. There were patients in all groups who responded with small advancements and on the other hand, patients who required larger protrusion to visualize improvement of the collapsibility.

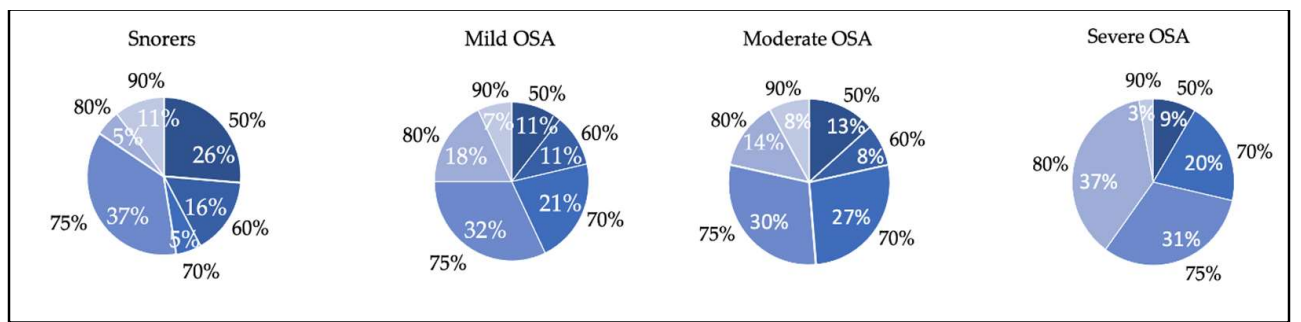

Figure 6: Percentages of advancement (in black) to obtain response to mandibular advancement according to the severity of the respiratory disorder. In all cases, the percentage of patients (in white) who responded to each percentage of advancement in each severity group is shown.

\section{Discussion}

To our knowledge, this is the first study that evaluates a combination of DISE with a manually controlled titratable mandibular positioner, SAM, as a prediction tool for oral appliance treatment outcome. This study arose in the high interindividual variability in performing jaw thrust maneuver during DISE, the great variability in response to the MAD and the need, not only to identify responders, but also to propose different recommendations according to the expected degree of response (SAM recommendations).

Our results after the DISE-SAM protocol suggest that MAD therapy could be recommended to $73.9 \%$ of the patients including S, A and $\mathrm{M}$ recommendations (see table 1). Our results expect total or partial response in 53,5\% (S and A recommendation) of the patients. For Sutherland et al. [14], 64\% of patients using MAD had total or partial responses in a meta-analysis. Metha et al., [13] found similar results (complete or partial response) in $62.5 \%$. The differences in these percentages with our results may be caused by the higher number of severe and moderate cases compared to snorers and mild patients in our study. According to the proposed SAM recommendation, in $19.3 \%$ of the cases, MAD could be used as single therapy (S recommendation) expecting a complete response. In $34.2 \%$ of patients, it was proposed as an alternative therapy (A recommendation), in which partial response was expected. And finally, for $20.5 \%$ of patients ( $\mathrm{M}$ recommendation) it was recommended only in combination with selected therapies as it could provide a synergistic effect. MAD was not recommended in $26.1 \%$ of patients. This value was slightly lower than the non-responders mentioned in Sutherland's meta-analysis [14] or Metha's study [13], who stated that $36 \%$ and $37.5 \%$ were non-responders. Again, this difference may be 
due to the incorporation of the new recommendation $(\mathrm{M})$ for some patients who would benefit from multiple therapies for the first time considered. In other words, with this DISE-SAM protocol, and proposing MAD YES/MAD NO, different levels of recommendation may be suggested, increasing the quality of the therapeutic recommendation.

In addition, the different SAM recommendations showed no relationship with severity. According to our results, MAD could be proposed for severe OSA in $9.3 \%$ of patients as an $\mathrm{S}$ recommendation, $25.9 \%$ as an A recommendation, and $29.6 \%$ as an $\mathrm{M}$ recommendation. In this view, $64.8 \%$ of severe OSA patients could benefit from MAD therapy. Perhaps, the recommendation based on a single variable, in this case, severity, is insufficient. Our observation suggests that the recommendation of MAD based on the severity of OSA is not accurate, as in all the severity groups, recommendations from $S$ to NO could be made (figure 4).

Thus, the DISE-SAM protocol could also be useful to personalize each responder's mandibular advancement regardless of severity. Our results found a positive relationship between the advancement required to obtain response and severity. However, this relation was weak, given the low value for the statistic test. Finally, we found patients in all severity groups with good responses to minor advancements, and others where maximal advancements were necessary to resolve the collapses (see Figure 6). MAD treatment could be no tolerable for these patients, with the necessary advancement being the largest, defining in advance that some patients would not be good candidates for this therapy. On the other hand, it has been reported that up to $39 \%$ of patients may respond with any advancement [42], and that in one third of patients, protruding the tongue or closing the mouth worsens the collapsibility [43]. Also, some results show that larger advancement is not always associated with a corresponding reduction in $\mathrm{AHI}[44,45]$. Knowledge of these aspects is very useful in treatment decision making.

Likewise, there is no consensus on oral appliance starting protrusive position (position in which the MAD is manufactured and delivered for subsequent gradual adaptation) nor on the recommended therapeutic position. This is due to high individual variability between practitioners for the former $[10,46,47]$ and a high inter-individual variability in response to the MAD therapy for the latter. Therefore, having information on the advancement required to alleviate the collapsibility, would be helpful in planning the starting point of advancement and the target position to be reached.

Studies looking at the effect of mandibular advancement during DISE [48-51] performed manual jaw thrust maneuvers with some authors [34-37] observing that the effect on UA of a mandibular hyperprotrusion or chin lift maneuvers do not always correlate with the results obtained with a MAD. It has been demonstrated that jaw thrust shows a greater improvement at hypopharyngeal level, but it is less effective in improving the obstruction at retropalatal level than a MAD [37]. However, the same authors [52] also concluded that both manual jaw thrust and a temporary MAD seem to mimic the treatment with a MAD. These authors focused on the total extent of obstruction release as potential predictor for treatment success. According to our findings, for an $\mathrm{M}$ recommendation, it is essential to identify the most similar expected response in every VOTE level to be as precise as possible for making the recommendation.

Similarly, a dental impression with the maximum comfortable protrusive for the patient has been recommended as advancement maneuver during sedation [34,35,53]. In addition to holding the mandible to prevent its dislodgement during the entire test, several simulation bites must be removed and reinserted from the mouth to assess changes when introducing different advancements. Performing these manipulations could cause stimulation of the patient that may produce arousals. Also, a remotely controlled mandibular positioner has been used during sedation to determine the effective target protrusive position within 45 minutes [54]. The authors found some limitations using this device: for some patients; it failed to register the full range of mandibular movement, the trays suffered slippage due to the forces exerted by the mandible losing the anchorage and slow 
continuous protrusion was not possible. Possibly these limitations were since this device was not intended for use in sedated patients.

By contrast, with the SAM device, the interexaminer bias of the jaw thrust maneuver is eliminated. The jaw can be moved manually forward or backward as slow (to avoid potential arousals) or as fast as needed from zero to maximum tolerable protrusion without being removed from the patients'mouth. All examinations could be performed regardless of the range of anteroposterior movement as its amplitude ranges between -20 to $+20 \mathrm{~mm}$, sufficient to adapt to any anatomy and mandibular mobility. While UA behavior is analyzed, the device maintains a fixed chosen mandibular position in supine or lateral decubitus. The upper and lower trays can be removed independently if needed or in an emergency to leave the airway free. Nevertheless, DISE was uneventful in our patients with no need for the SAM anticipated extraction. In addition, with the SAM, DISE can be performed in 15-20 minutes, thus reducing examination time.

Following our protocol, the SAM reproduces the minimum vertical dimension needed to use a MAD. For some authors patient's preference was clearly in favor of the lower vertical dimension wearing a MAD [55] as a higher dimension favors jaw discomfort and may increase collapsibility of the UA [56]. During our protocol, we did have in mind that increasing the sheer size also tends to a posterior rotation causing a $0,3 \mathrm{~mm}$ reduction in the range of mandibular advancement for every $1 \mathrm{~mm}$ of vertical opening [57]. Therefore, the mandibular protrusion capacity should not be limited as it is essential for a successful treatment for most patients [13,58].

Despite the fact some authors $[59,60,61]$ concluding that functional endoscopy is an optimal predictor for MAD treatment outcome it could be controversial since it investigates the dynamic behavior of the upper airway during wakefulness. In addition, Okuno et al. [59] ,60] only focus on the cross-sectional area changes at the velopharinx to make the recommendation. According to some publications [62-64] DISE has several advantages including safety, ease of use, and reliability, which outweigh awake exploration to diagnose sites of obstruction and the pattern of UA collapse. It also has strong informative evidence on changes in the upper airway when introducing mandibular advancement maneuvers.

This study is no exception in having limitations. First, this work investigates the effect of MAD on DISE, not during natural sleep. By using propofol, we could not reproduce REM sleep. Second, a follow-up study of patients treated with MAD after this DISE-SAM protocol is required to verify the success of the recommendation. Third, in the future research agenda, it is mandatory to confirm with control sleep studies the utility of DISESAM protocol to select patients for MAD treatment and verify the usefulness of this technique in the selection of a combination of therapies.

\section{Conclusions}

The SAM device allowed a reproducible, measurable, and titratable maneuver to maintain a stable position during DISE. Given that there was no relationship between OSA severity and SAM recommendation and a weak relationship between severity and the required advancement, therefore a precise mandibular maneuver during DISE is needed to accurately identify the potential responders.

Using the DISE-SAM protocol the response to mandibular advancement under sedation regardless of severity can be identified. The protocol also allows measuring the range of mandibular protrusion in which improvement of UA collapsibility could be achieved.

Therefore, the selection of therapeutic strategies should be a highly individualized process. According to SAM recommendations, MAD can be proposed within the therapeutic armamentarium for sleep-disordered breathing in the most personalized design as a single, alternative, or multiple treatment approaches.

Supplementary Materials: The following are available online at Video S1: Supplementary Video. 
Author Contributions: Conceptualization, P.F-S.; J.A.; J.S.; M.A.; G.B.; N.P.-M.; A.P. and G.P.; methodology, P.F-S..; software, P.F-S..; validation, P.F-S.; J.A.; J.S.; M.A.; G.B.; N.P.M. and A.P.; formal analysis, P.F-S.; M.R.L.; investigation, P.F-S.; resources, P.F-S.; data curation, P.F-S.; J.A.; J.S.; M.A.; G.B.; N.P.-M. and A.P..; writing-original draft preparation, P.F-S.; writing-review and editing, P.F-S.; M.R-L. and G.P.; visualization, P.F-S.; N.P.-M.; M.C.-L1.; I. M.-H.; R.L.; C.O-R., P.B. and G.P.; supervision, P.F-S.; C.O-R., P.B. and G.P.; project administration, P-F-S. and G.P. All authors have read and agreed to the published version of the manuscript.

Funding: This research received no external funding.

Institutional Review Board Statement: The study was conducted according to the guidelines of the Declaration of Helsinki. The study protocol was approved by the Internal Review Board of the Hospital Universitario de Fuenlabrada. APR 20/30.

Informed Consent Statement: Informed consent was obtained from all subjects involved in the study.

Data Availability Statement: Data are contained within the article.

Acknowledgments: The authors are grateful for the technical support of the Sleep Unit and the library service of the Hospital Universitario de Fuenlabrada.

Conflicts of Interest: Patricia Fernández-Sanjuán is the creator of SAM device. Juan José Arrieta, Jaime Sanabria, Marta Alcaraz, Gabriela Bosco, Nuria Pérez-Martín, Adriana Pérez, Marina Carrasco-LLatas, Isabel Moreno-Hay, Marcos Ríos-Lago, Rodolfo Lugo, Carlos O'Connor-Reina, Peter Baptista, and Guillermo Plaza declare no conflicts of interest regarding the publication of this paper.

$\begin{array}{ll}\text { Abbreviations } \\ \text { MAD } & \text { Mandibular advancement device } \\ \text { DISE } & \text { Drug induced sleep endoscopy } \\ \text { SAM } & \text { Selector avance mandibular } \\ \text { OSA } & \text { Obstructive sleep apnea } \\ \text { CPAP } & \text { Continuous positive airway pressure } \\ \text { AHI } & \text { Apnea-hypopnea index } \\ \text { BMI } & \text { Body mass index } \\ \text { UA } & \text { Upper airway } \\ \text { BIS } & \text { Bispectral index }\end{array}$

\section{References}

1. Heinzer, R.; Vat, S.; Marques-Vidal, P.; Marti-Soler H, Andries, D.; Tobback, N.; Mooser, V.; Preisig, M.; Malhotra, A.; Waeber, G.; Vollenweider, P.; Tafti, M.; Haba-Rubio, J. Prevalence of sleep-disordered breathing in the general population: the HypnoLaus study. Lancet Respir. Med. 2015, 3, 310-8.

2. Tietjens, J.R.; Claman, D.; Kezirian, E.J.; De Marco, T.; Mirzayan, A.; Sadroonri, B.; Goldberg, A.N.; Long, C.; Gerstenfeld, E.P.; Yeghiazarians, Y. Obstructive Sleep Apnea in Cardiovascular Disease: A Review of the Literature and Proposed Multidisciplinary Clinical Management Strategy. J. Am. Heart Assoc. 2019, 8, 1. 
3. Allen, A.J.M.H.; Bansback, N.; Ayas, N.T. The effect of OSA on work disability and work-related injuries. Chest 2015, 147,14228.

4. Richard, W.; Venker, J.; den Herder, C.; Kox, D.; van den Berg, B.; Laman, M.; van Tinteren, H.; de Vries, N. Acceptance and Long-Term Compliance of CPAP in Obstructive Sleep Apnea. Eur. Arch. Otorhinolaryngol. 2007, 264, 1081-6.

5. Weaver, T.E.; Grunstein, R.R. Adherence to Continuous Positive Airway Pressure Therapy: The Challenge to Effective Treatment. Proc. Am. Thorac. Soc 2008, 5, 173-8.

6. Hamoda, M.M.; Kohzuka, Y.; Almeida, F.R. Oral Appliances for the Management of OSA: An Updated Review of the Literature. Chest 2018, 153, 544-53.

7. Ferguson, K.A.; Ono, T.; Lowe, A.A.; Al-Majed, S.; Love, L.L.; Fleetham J.A. A short-term controlled trial of an adjustable oral appliance for the treatment of mild to moderate obstructive sleep apnoea. Thorax 1997, 52, 362-8.

8. Sutherland, K.; Vanderveken, O.M.; Tsuda, H.; Marklund, M.; Gagnadoux, F.; Kushida, C.A.; Cistulli, P.A. on behalf of the ORANGE-Registry (Oral Appliance Network on Global Effectiveness). Oral Appliance Treatment for Obstructive Sleep Apnea: An Update. J. Clin. Sleep Med. 2014, 10, 215-27.

9. Bloch, K.; Iseli, A.; Zhang, J.N.; Xie, X.; Kaplan, V.; Stoeckli, P.W.; Russi, E.W. A randomized, controlled crossover trial of two oral appliances for sleep apnea treatment. Am. J. Respir. Crit. Care Med. 2000, 162, 246-51.

10. Dieltjens, M.; Vanderveken, O.M.; Van de Heynin, P.H.; Braem, M. Current Opinions and clinical practice in the titration of oral appliances in the treatment of sleep disordered breathing. Sleep Med. Rev. 2012, 16, 177-85.

11. Ramar, K.; Dort, L.C.; Katz, S.G.; Lettieri, C.J.; Harrod, C.G.; Thomas, S.M.; Chervin, R. D. Clinical practice guideline for the treatment of obstructive sleep apnea and snoring with oral appliance therapy: an update for 2015. J. Clin. Sleep Med. 2015, 11, 773-827.

12. Chan, A.S.; Cistulli, P.A. Oral appliance treatment of obstructive sleep apnea: an update. Curr. Opin. Pulm. Med. 2009, 15, 5916.

13. Mehta, A.; Qian, J.; Petocz, P.; Darendeliler, M.A.; Cistulli, P.A. A randomized controlled study of a mandibular advancement splint for obstructive sleep apnea. Am. J. Respir. Crit. Care Med. 2001, 163, 1457-61.

14. Sutherland, K.; Takaya, H.; Qian, J.; Petocz, P.; Ng, AT.; Cistulli P.A. Oral Appliance Treatment Response and Polysomnographic Phenotypes of Obstructive Sleep Apnea. J. Clin. Sleep Med. 2015, 11, 861-8.

15. Ferguson, K.A.; Cartwright, R.; Rogers, R.; Schmidt-Nowara, W. Oral Appliances for Snoring and Obstructive Sleep Apnea: A Review. Sleep 2006, 29, 244-62.

16. Dieltjens, M.; Braem, M.J.; Van de Heyning, P.H.; Wouters, K.; Vanderveken, O.M. Prevalence and clinical significance of supine-dependent obstructive sleep apnea in patients using oral appliance therapy. J. Clin. Sleep Med. 2014, 10, 959-64.

17. Takaesu, Y.; Tsuiki, S.; Kobayashi, M.; Komada, Y.; Nakayama, H.; Inoue, Y. Mandibular advancement device as a comparable treatment to nasal continuous positive airway pressure for positional obstructive sleep apnea. J. Clin. Sleep Med. 2016, 12,11139.

18. Petri, N.; Svanholt, P.; Solow, B.; Wildschiødtz, G.; Winkel, P. Mandibular advancement appliance for obstructive sleep apnoea: results of a randomised placebo-controlled trial using parallel group design. J. Sleep Res. 2008, 17, 221-9.

19. Holley, A.B.; Lettieri, C.J.; Shah, A.A. Efficacy of an adjustable oral appliance and comparison with continuous positive airway pressure for the treatment of obstructive sleep apnea syndrome. Chest 2011, 140, 1511-6.

20. Lettieriet, C.J.; Paolino, N.; Eliasson, A.H.; Shah, A.A.; Holley, A.B. Comparison of adjustable and fixed oral appliances for the treat- ment of obstructive sleep apnea. J. Clin. Sleep. Med. 2011, 7, 439-45.

21. Liu, Y.; Lowe, A.A.; Fleetham, J.A.; Park, Y.C. Cephalometric and physiologic predictors of the efficacy of an adjustable oral appliance for treating obstructive sleep apnea. Am. J. Orthod. Dentofacial Orthop. 2001, 120, 639-47.

22. Marklund, M.; Stenlund, H.; Franklin, K.A. Mandibular advancement devices in 630 men and women with obstructive sleep apnea and snoring: tolerability and predictors of treatment success. Chest 2004, 125, 1270-8.

23. Denolf, P.L.; Vanderveken, O.M.; Marklund, M.E.; Braem, M.J. The status of cephalometry in the prediction of non-CPAP treatment outcome in obstructive sleep apnea patients. Sleep Med. Rev. 2016, 27, 56-73.

24. Tsuiki, S.; Kobayashi, M.; Namba, K.; Oka, Y.; Komada, Y.; Kagimura ,T. Optimal positive airway pressure predicts oral appliance response to sleep apnoea. Eur. Respir. J. 2010, 35, 1098-105.

25. Storesund, A.; Johansson, A.; Bjorvatn, B.; Lehmann, S. Oral appliance treatment outcome can be predicted by continuous positive airway pressure in moderate to severe obstructive sleep apnea. Sleep Breath. 2018, 22, 385-92.

26. Sutherland, K.; Phillips, C.L.; Davies, A.; Srinivasan, V.K.; Dalci, O.; Yee, B.J.; Darendeliler, M.A.; Grunstein, R.R.; Cistulli, P.A. CPAP Pressure for Prediction of Oral Appliance Treatment Response in Obstructive Sleep Apnea. J. Clin. Sleep Med. 2014, 10, 943-9.

27. Ecker, D.J. Phenotypic approaches to obstructive sleep apnoea - New pathways for targeted therapy. Sleep Med. Rev. 2018, 37, 45-59.

28. Edwards, B.A.; Andara, C.; Landry, S.; Sands, S.A.; Joosten, S.A.; Owens, R.L.; White, D.P.; Hamilton, G.S.; Wellman, A. UpperAirway Collapsibility and Loop Gain Predict the Response to Oral Appliance Therapy in Patients with Obstructive Sleep Apnea. Am. J. Respir. Crit. Care Med. 2016, 194, 1413-22.

29. Op de Beeck, S.; Dieltjens, M.; Verbruggen, A.E.; Vroegop, A.V.; Wouters, K.; Hamans, E.; Willemen, M.; Verbraecken, J.; De Backer, W.A.; Van de Hevning, P.H; Braem, M.J.; Vanderveken, O.M. Phenotypic Labelling using Drug-Induced Sleep 
Endoscopy Improves Patient Selection for Mandibular Advancement Device Outcome: A prospective study. J. Clin. Sleep Med. 2019, 15, 1089-99.

30. Sutherland, K.; Chan, A.S.L.; Ngiam, J.; Dalci, O.; Darendeliler, M.A.; Cistulli, P.A. Awake Multimodal Phenotyping for Prediction of Oral Appliance Treatment Outcome. J. Clin. Sleep Med. 2018, 14, 1879-87.

31. De Vito, A.; Carrasco Llatas, M.; Vanni, A.; Bosi, M.; Braghiroli, A.; Campanini, A.; de Vries, N.; Hamans, E.; Hohenhorst, W.; Kotecha, B.T.; Maurer, J.; Montevecchi, F.; Piccin, O.; Sorrenti, G.; Vanderveken, O.M.; Vicini, C. European position paper on drug-induced sedation endoscopy (DISE). Sleep Breath. 2014, 18, 453-65.

32. Carrasco Llatas,M.; Dalmau Galofre, J.; Zerpa Zerpa, V.; Marcano Acuña. M.; Mompó Romero, L. Drug-induced Sleep Videoendoscopy: Clinical Usefulness and Literature Review. Acta Otorrinolaringol. Esp. 2014, 65, 183-90.

33. Croft, C.B.; Pringle, M. Sleep nasoendoscopy: a technique of assessment in snoring and obstructive sleep apnoea. Clin. Otolaringol. Allied Sci. 1991, 16, 504-9.

34. Vanderveken, O.M.; Vroegop, A.M.; van de Heyning,P.H.; Braem, M.J. Drug-induced sleep endoscopy completed with a simulation bite approach for the prediction of the outcome of treatment of obstructive sleep apnea with mandibular repositioning appliances. Op. Tech. Otolaryngol. Head Neck Surg. 2011, 22, 175-182.

35. Vroegop, A.V.; Vanderveken, O.M.; Dieltjens, M.; Wouters, K.; Saldien, V.; Braem, M.J.; Van de Heyning, P.H. Sleep endoscopy with simulation bite for prediction of oral appliance treatment outcome. J. Sleep Res. 2013, 22, 348-55.

36. Vonk, P. E., Beelen, A., de Vries, N. Towards a prediction model for drug-induced sleep endoscopy as selection tool for oral appliance treatment and positional therapy in obstructive sleep apnea. Sleep Breath. 2018, 22, 901-907.

37. Vonk, P.E.; Uniken Venema, J.A.M.; Hoekema, A.; Ravesloot, M.J.L.; van de Velde, J.A.; de Vries, N. Jaw thrust Versus the Use of a Boil-And-Bite Mandibular Advancement Device as a Screening Tool During Drug-Induced Sleep Endoscopy. J. Clin. Sleep Med. 2020, 16, 1021-7.

38. Park, D.; Kim, J-SS.; Heo, S.J. The Effect of the Modified Jaw-Thrust Maneuver on the Depth of Sedation During Drug-Induced Sleep Endoscopy. J. Clin. Sleep Med. 2019, 15, 1503-8.

39. Berry, R.B.; Budhiraja, R.; Gottlieb, D.J.; Gozal, D.; Iber, C.; Kapur, V.K.; Marcus, C.L.; Mehra, R.; Parthasarathy, S.; Quan, S.F.; et al. Rules for scoring respiratory events in sleep: Update of the 2007 AASM Manual for the Scoring of Sleep and Associated Events. Deliberations of the Sleep Apnea Definitions Task Force of the American Academy of Sleep Medicine. J. Clin. Sleep Med 2012, 15, 597-619.

40. Kezirian, E.J.; Hohenhorst, W.; de Vries, N. Drug-induced Sleep Endoscopy: The VOTE Classification. Eur. Arch. Otorhinolaryngol. 2011, 268, 1233-36.

41. Vonk, P.E.; van de Beek, M.J.; Ravesloot, M.J.L.; de Vries, N. Drug-induced sleep endoscopy: new insights in lateral head rotation compared to lateral head and trunk rotation in (non)positional obstructive sleep apnea patients: DISE: Lateral Head (and Trunk) Rotation. Laryngoscope 2019, 129, 2430-5.

42. Anitua, E.; Durán-Cantolla, J.; Almeida, G.Z.; Alkhraisat, M.H.; Minimizing the mandibular advancement in an oral appliance for the treatment of obstructive sleep apnea. Sleep Med. 2017, 34, 226-31.

43. Bonzelaar, L.B.; Salapatas, A.M.; Hwang, S.M.; Andrews, C.C.; Price, N.Y.; Friedman, M. The Effect of Oral Positioning on the Hypopharyngeal Airway. Laryngoscope 2017, 127, 1471-5.

44. Ng Andrew, T.; Gotsopoulos, H.; Qian, J.; Cistulli PA. Effect of oral appliance therapy on upper airway collapsibility in obstructive sleep apnea. Am. J. Respir. Crit. Care Med. 2003, 168, 238-41.

45. Bartolucci, M.L.; Bortolotti, F.; Raffaelli, E. The effectiveness of different mandibular advancement amounts in OSA patients: a systematic review and meta-regression analysis. Sleep Breath. 2016, 20, 911-9.

46. Ngiam, J.; Balasubramaniam, R.; Darendeliler, M.A.; Cheng, A.T.; Waters, K.; Sullivan, C.E. Clinical guidelines for oral appliance therapy in the treatment of snoring and obstructive sleep apnoea. Aust. Dent. J. 2013, 58, 408-19.

47. Gindre, L.; Gagnadoux, F.; Meslier, N.; Gustin, J.M.; Racineux, J.L. Mandibular advancement for obstructive sleep apnea: dose effect on apnea, long-term use and tolerance. Respiration 2008, 76, 386-92.

48. Johal, A.; Battagel, J.M.; Kotecha, B.T. Sleep nasendoscopy: a diagnostic tool for predicting treatment success with mandibular advancement splints in obstructive sleep apnoea. Eur. J. Orthod. 2005, 27, 607-14.

49. Johal, A.; Hector, M.P.; Battagel, J.M.; Kotecha, B.T. Impact of sleep nasendoscopy on the outcome of mandibular advancement splint therapy in subjects with sleep-related breathing disorders. J Laryngol Otol. 2007, 121, 668-75.

50. Eichler, C.; Sommer, J.U.; Stuck, B.A.; Hörmann, K.; Maurer, J.T. Does drug-induced sleep endoscopy change the treatment concept of patients with snoring and obstructive sleep apnea. Sleep Breath. 2013, 17, 63-8.

51. De Corso, E.; Bastanza, G.; Della Marca, G.; Grippaudo, C.; Rizzoto, G.; Marchese, M.R.; Fiorita, A.; Sergi, D.; Meucci, D.; Di Nardo, W.; Paludetti, G.; Scarano, E. Drug-induced sleep endoscopy as a selection tool for mandibular advancement therapy by oral device in patients with mild to moderate obstructive sleep apnoea. Acta Otorhinolarynngol. Ital. 2015, 35, 426-3.

52. Bosschieter, P.F.N.; Vonk, P.E.; de Vries, N. The predictive value of drug-induced sleep endoscopy for treatment success with a mandibular advancement device or positional therapy for patients with obstructive sleep apnea. Sleep Breath. 2021 , Oct 1.

53. Cavaliere, M., De Luca, P.; De Santis, C.; Scarpa, A.; Ralli, M.; Di Stadio, A.; Viola, P.; Chiarella, G.; Cassandro, C.; Cassandro, F. Drug-induced Sleep Endoscopy (DISE) with Simulation Bite to Predict the Success of Oral Appliance Therapy in Treating Obstructive Sleep Apnea/Hypopnea Syndrome (OSAHS). Translational medicine @ UniSa 2020, 23, 58-62.

54. Kastoer, C.; Dieltjens, M.; Op de Beeck, S.O.; Braem, M.J.; Van de Heyning, P.H.; Vanderveken, O.M.; Remotely Controlled Mandibular Positioning During Drug-Induced Sleep Endoscopy Toward Mandibular Advancement Device Therapy: Feasibility and Protocol. J. Clin. Sleep Med. 2018, 14, 1409-13. 
55. Pitsis, A.J.; Darendeliler, M.A.; Gotsopoulos, H.; Petocz, P.; Cistulli, P.A. Effect of Vertical Dimension on Efficacy of Oral Appliance Therapy in Obstructive Sleep Apnea. Am J Respir Crit Care Med 2002, 166, 860-4.

56. Vroegop, A. V.; Vanderveken, O. M; Van de Heyning, P. H., Braem, M. J. Effects of vertical opening on pharyngeal dimensions in patients with obstructive sleep apnoea. Sleep Med 2012, 13, 314-6.

57. Mayoral, P.; Lagravère, M.O.; Garcia, M. Antero-posterior mandibular position at different vertical levels for mandibular advancing device design. BMC Oral Health 2019, 19-85.

58. Remmers, J.; Charkhandeh, S.; Grosse, J.; Topor, Z.; Brant, R.; Santosham, P.; Bruehlmann, S. Remotely controlled mandibular protrusion during sleep predicts therapeutic success with oral appliances in patients with obstructive sleep apnea. Sleep 2013 $36,1517-25,1525 \mathrm{~A}$.

59. Okuno, K.; Sasao, Y.; Nohara, K.; Sakai, T.; Pliska, B.T.; Lowe, A.A.; Ryan, C.F.; Almeida, F.R. Endoscopy evaluation to predict oral appliance outcomes in obstructive sleep apnoea. Eur. Respir. J. 2016, 47, 1410-1419.

60. Okuno, K.; Ikai, K.; Matsumura-Ai, E.; Araie, T. Titration technique using endoscopy for an oral appliance treatment of obstructive sleep apnea. J. Prosthet. Dent. 2018, 119, 350-353.

61. Gasparini, G.; Saponaro, G.; Todaro, M.; Ciasca, G.; Cigni, L.; Doneddu, P.; Azzuni, C.; Foresta, E.; De Angelis, P.; Barbera, G., Parcianello, R. G.; Hreniuc, H. V.; Moro, A. Functional Upper Airway Space Endoscopy: A Prognostic Indicator in Obstructive Sleep Apnea Treatment with Mandibular Ad-vancement Devices. Int J Environ Res Public Health. 2021, 18, 2393.

62. Soares D.; Folbe A.J.; Yoo, G.; Badr, M.S.; Rowley, J.A.; Lin, H.S. Drug-induced sleep endoscopy vs awake Müller's maneuver in the diagnosis of severe upper airway obstruction. Otolaryngol Head Neck Surg. 2013, 148, 151-6.

63. Cavaliere, M.; Russo, F.; Iemma, M.; Awake versus drug-induced sleep endoscopy: evaluation of airway obstruction in obstructive sleep apnea/hypopnoea syndrome. Laryngoscope. 2013, 123, 2315-8.

64. Yegïn, Y.; Çelik, M.; Kaya, K.H.; Koç, A.K.; Kayhan, F.T.Comparison of drug-induced sleep endoscopy and Müller's maneuver in diagnosing obstructive sleep apnea using the VOTE classification system. Braz J Otorhinolaryngol. 2017, 83, 445-450. 